\title{
Güler and Linaro et al Model in an Investigation of the Neuronal Dynamics using noise Comparative Study
}

\author{
Renas Rajab Asaad \\ Department of Computer Science, Nawroz University, Duhok, Kurdistan Region - Iraq
}

\begin{abstract}
Recently, theoretical arguments, numerical simulation and experiments shown that ion channel noise in neurons can have deep impact on the behavior of the neuron's dynamical when there is a limited size for the membrane space. It can be create different models of Linaro al equations by using stochastic differential equations to find the impacts of ion channel noise, and it has been analytically put forward the Güler model. More recently, Güler has discussed that in small neurons the rate functions for the closing and opening of gates are under the effect of the noise. In this research, the investigation of dynamics neurons are determined with noise rate functions. The exact Markov simulations will be employ during the investigation with above analytical models. Comparatively, the results will be presented from these models. The research aims to show more details on the phenomenon recently outlined by Güler.
\end{abstract}

Keywords: Guler Model, Linaro et al Model, Neural Network, Neuron Science.

\section{Introduction}

The influence of noise to the neurons generates an abnormal prototype on the neuronal dynamics. The noise is in two categories; internal or external [3]. External noise is the contradictory of internal. External noise is generated from the synaptic signal transmission. The main source of internal noise in a neuronal membrane stain is from the finite number of voltage-gates ion's channels. Commonly; these channels has two cases; open or close. When the case being consider open, the channels' fluctuations number are randomly present [5]. Neurons show electrical activity which is identified to be stochastic in distribution [3]. The main source of stochasticity in vivo is the external noise from the synapses. Nevertheless, the essential noise, characterized to the potential attribute of an ion channel shift, may has important inclusion on the dynamic.

The dynamic activities of neurons has been expressed by both experimental studies $[5,8,9]$. Default spiking is an incident happen by the internal noise from ion channels. There is a theoretic investigations or arithmetic simulations of channel dynamics (in the case of recurrence spiking and bursting), or else noiseless

Academic Journal of Nawroz University (AJNU) Volume

8, No 3 (2019).

Regular research paper : Published 11 June 2019

Corresponding author's e-mail : renas_rekany@yahoo.com

Copyright @2018 Renas Rajab Asaad.

This is an open access article distributed under the

Creative Commons Attribution License. membrane spots [9];

Moreover, these investigations also have unhidden occurrence of stochastic echo and the coherence of the created spike trains $[4,6,8]$. When the number of ion channels is big means the membrane size is big too, the voltage dynamics will reduce as in the prime Linaro et al [11]. It's called dissipative stochastic mechanics (it's shortcut of DSM) had been put up by Guler, renormalization improvements expand the activity of transformations from low to higher spiking (Jibril and Güler 2009). Also, DSM model in the case of variable time input streams.

\section{Structures of Neurons}

The neuron is the main structure of nervous tissue. It is a very specialized cell that varies in size, length and shape. It may range from a few millimeters to a few meters, like in a whale. It is found in parts of the main nervous system (brain, spinal cord) In the human body while its axes are scattered in the different parts of the body and are characterized by transport and transport are carried in one direction from the neurotic to the body of the cell and the body of the cell to the axis nerve. The neuron is not manipulated because humans generate a tumor in all its nerve cells, stop dividing before or when they are formed, and thus enter the numbers of permanent cells that do not divide. If nerve cells are damaged, we create a new neuronal cell to 
replace them. As shown in the figure below it is composed. The neuron of the following:

1. Cell Body: The body of the cell contains the cytoplasm, nucleus, collagen, mitochondria, chromosome, and a network of neural fibers, as well as other RNA fibers and materials in the form of granules with bodies or granules of offspring. They are composed of thin, The cell is related to the neuronal activities of the cell, and the neuron does not contain the centrolite, so it loses its ability to divide.

2. Dendrites: Is stand out from the body of the neuron, there are polygons that vary from cell to cell. They may have one branch called a single pole cell. Two branches, called a bipolar cell, are called multiple poles.

3. Axon: An axon, or nerve fiber, is a long, slender projection of a nerve cell.

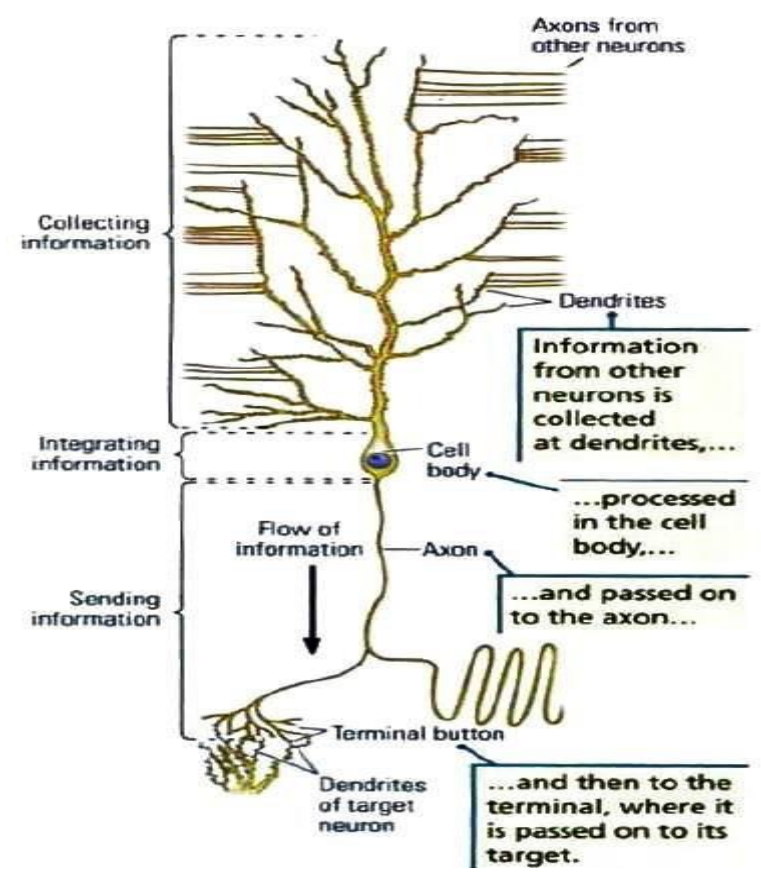

Fig 1: Two Interconnected Cortical Pyramidal Neurons [7]

\section{A. Spike}

Spike neural networks (SNNs) are models of artificial neural networks that resemble natural neural networks more closely [7].

\section{B. Membrane proteins}

Membrane proteins are protein molecules that are associated with or associated with the living membranes of the living cells or the kidneys.. These three groups help to passing potassium and calcium through the membrane. These three groups are: channels, gates, and pumps.

\section{Channels}

Channels created by some membranes proteins and its allow to pass one of sodium and potassium through channels.

\section{Gates}

The form of gates change according to it-self, It will be active when the key match with the embedded proteins by the size and its form.

\section{Pumps}

Among the substances that are transported by primary transport agent are sodium, potassium, calcium, hydrogen, chlorine and many other electrolytes. However, all of these substances are not transferred through the membranes of all cells by active transport. Moreover, some transfer functions occur at the membrane inside the cell, not (or in addition to) the outer surface of the cell, as in the membrane of the musculoskeletal network or mitochondrial membrane. However, it works essentially with the same basic mechanism.

\section{Neuron Electrical Activity and Membrane Potential}

The electrical signals resulting from brain cell reactions reflect the state of arousal of certain parts in it. This state of affairs is the result of different brain reactions whose causes are due to patterns of thought, emotion, behavior, movement and sensation. For example, when a person puts his hand on a hot stove, the autonomic nervous system first reacts by immediately removing the hand from the burner before he becomes aware of the injury. Thus, an electrical signal is sent through the spinal cord (Thalamus), which in turn sends it to specific parts of the brain such as the somatosensory cortex, which is responsible for somatosensory cortex, the frontal cortex, Limbic system. In the end, he feels the pain in the hand, thinks about what happened to him, and emotionally reacts according to the intensity of the pain.

All parts of the brain work together, each part of which is responsible for a specific function, ranging from heart rate to mood. All these functions trigger multiple electrical activities between the brain cells, and because of these, brain waves are readable through electrodes Which are placed on the scalp as we mentioned the first part of this series. Each wave has characteristics that differ from those of other waves. These properties are the frequency and amplitude of the wave. They 
determine the type of vector (Delta, Alpha, Beta, Eutha, or Gamma).

As we have mentioned, Are different in humans and are stimulated by different places in the brain. These waves have been discovered for various reasons. Researchers can, by reading them, determine when they occur and can also link this information to external and internal influences. [2]. Figure 2 discuss about the dynamic voltages cases of passing ions (potassium and sodium).

The fig (2) shows the resting potential in real value $70 \mathrm{mV}$.

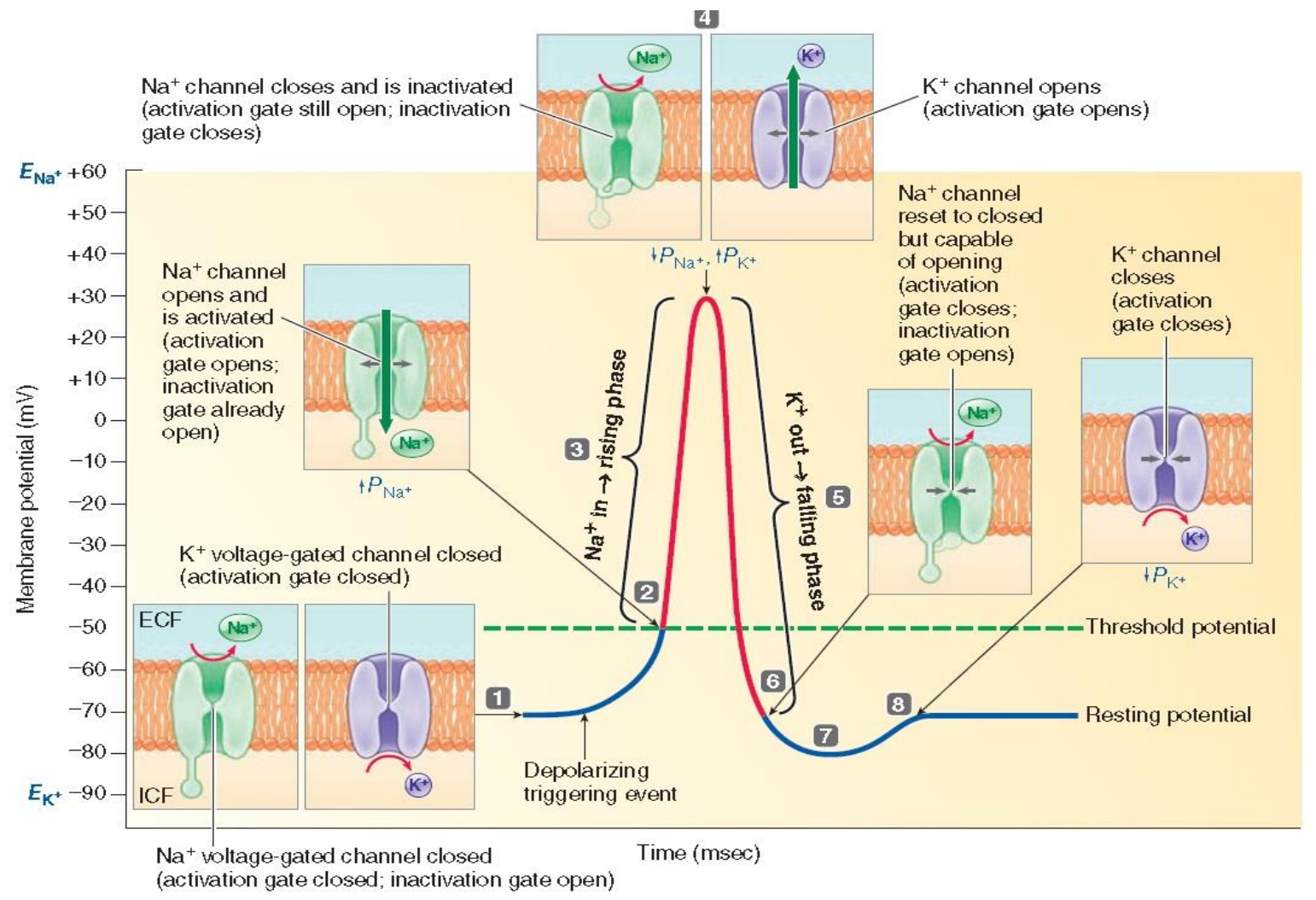

Fig 2: Phases of action potential [12]

\section{The Linaro et al. Model}

In the linaro model uses the suitable powers of the variables of deterministic gating in determining the ratio of open channels; nevertheless, some processes of Ornstein-Uhlenbeck, with the diffusions acquired from the co-variances of $n^{4}$ and $m^{3} h$, escort the conductances. In consequence, the noise term escorting potassium conductance, $\emptyset_{K}^{L}$, as follow:

$$
\emptyset_{K}^{L}=\sum_{i=1}^{4} Z_{K, i}
$$

Where $Z_{K, i}$ are stochastic variables conforming:

$$
\tau_{K, i} \dot{Z}_{K, i}=-Z_{K, i}+\alpha_{K, i} \sqrt{2 \tau_{K, i} \xi_{K, i}}
$$

Where the coefficients $\tau_{K, i}$ and $\alpha_{K, i}$ are some functions of the closing and opening rates of $n$-gates (available in Linaro et al., 2011). And the $\xi_{K, i}$ are independent unitary variances and gaussian white noise is equal to zero. The noise term related with sodium channels, $\emptyset_{K}^{L}$ as follow:

$$
\emptyset_{N a}^{L}=\sum_{i=1}^{4} Z_{N a, i}
$$

Where $Z_{N a, i}$ are stochastic variables obey:

$$
\tau_{N a, i} \dot{Z}_{N a, i}=-Z_{N a, i}+\alpha_{N a, i} \sqrt{2 \tau_{N a, i} \xi_{N a, i}}
$$


Where the coefficients $\tau_{N a, i}$ and $\alpha_{N a, i}$ are some functions of the closing and opening rates of $\mathrm{m}$-gates and h-gates (available in Linaro2011). And the $\xi_{N a, i}$ are independent with zero means:

$$
\eta_{n}^{L}=\eta_{m}^{L}=\eta_{h}^{L}=0
$$

\section{Stochastic Model}

The dynamics of neurons under the influence of channel oscillation are usually designed with randomized differential equations obtained using some white noise conditions that fade in fundamental deterministic equations [6]. DSM neuron-based neurotransmitters raised by Güler 10], is a special case of this. The DSM model contains some functional shapes called recalibration conditions, as well as some white noise conditions that fade in activity equations. The DSM model has some functional shapes and a recalibration defect, to the extent of white noise conditions. The DSM model has been studied in numerical detail of time dynamics [9]. Renormalization patches have been shown to be changes in behavior from calm to spike and from bursting fire. as shown in experimental studies $[5,8,9]$ and by theoretical research or the numerical simulations [6]).

\section{A. The Güler Model}

No variables are placed to be zero in [10] model. The noise terms escorting the conductances $\emptyset_{N a}^{G}$ and $\emptyset_{K}^{G}$ were inserted to capture NCCP. Both terms $\emptyset_{N a}^{G}$ and $\emptyset_{K}^{G}$ are functions of the gating variables: the previous is dependent on $n, m$, and $h$. The term $\emptyset_{K}^{G}$, which reflects NCCP attributed to the potassium channels, is given by:

$$
\emptyset_{K}^{L}=\sqrt{\frac{n^{4}\left(1-n^{4}\right)}{N_{K}} q_{K}}
$$

$q_{K}$ is differential equation of stochastic,

$$
\begin{gathered}
\tau q_{\dot{K}=} p_{K} \\
\tau q_{\dot{K}=\gamma_{K}} p_{K}-\varpi_{K}^{2}\left[\alpha_{n}(1-n)+\beta_{n} n\right] q_{K}+\xi_{K}
\end{gathered}
$$

$\xi_{K}=$ zero gaussian white noise term,

$$
\begin{gathered}
\left(\xi_{K}(t) \xi_{K}\left(t^{\prime}\right)\right)=\gamma_{K} T_{K}\left[\alpha_{n}(1-n)+\beta_{n} n\right] \delta\left(t-t^{\prime}\right) \\
\gamma_{K}=10, \quad \varpi_{K}^{2}=150, \quad T_{K}=400 \\
\emptyset_{N a}^{L}=\sqrt{\frac{n^{4}\left(1-n^{4}\right)}{N_{N a}} q_{N a}}
\end{gathered}
$$

$q_{N a}$ is the differential equation of stochastic,

$$
\begin{gathered}
\tau \dot{q}_{N a}=p_{N a} \\
\tau \dot{q}_{N a}=\gamma_{N a} p_{N a}-\varpi_{N a}^{2}\left[\alpha_{n}(1-n)+\beta_{n} n\right] q_{N a}+\xi_{N a}
\end{gathered}
$$

$\xi_{N a}$ with the mean square is mean zero gaussian white noise term,

$$
\left(\xi_{N a}(t) \xi_{N a}\left(t^{\prime}\right)\right)=\gamma_{N a} T_{N a}\left[\alpha_{n}(1-n)+\beta_{n} n\right] \delta\left(t-t^{\prime}\right)
$$

$$
\gamma_{K}=10, \varpi_{K}^{2}=200, T_{K}=800
$$

Gaussian and the mean squares satisfy:

$$
\begin{aligned}
& \left(n_{n}(t) n_{n}\left(t^{\prime}\right)\right)^{G}=\frac{\alpha_{n}(1-n)+\beta_{n} n}{4 N_{K}} \delta\left(t-t^{\prime}\right) \\
& \left(n_{m}(t) n_{m}\left(t^{\prime}\right)\right)^{G}=\frac{\alpha_{m}(1-m)+\beta_{m} m}{3 N_{N a}} \delta\left(t-t^{\prime}\right) \\
& \left(n_{h}(t) n_{h}\left(t^{\prime}\right)\right)^{G}=\frac{\alpha_{h}(1-h)+\beta_{h} h}{N_{h}} \delta\left(t-t^{\prime}\right)
\end{aligned}
$$

These variances from the non-equilibrium statistical mechanics that the stochastic variables $\mathrm{q}_{\mathrm{K}}$ and $\mathrm{q}_{\mathrm{Na}}$ crop the following variances, respectively, at equilibrium:

$$
\left(q_{K}^{2}\right)_{e q}=\frac{T_{K}}{2 \varpi_{K}^{2}}
$$

And $\quad\left(q_{N a}^{2}\right)_{e q}=\frac{T_{N a}^{2}}{2 \varpi_{N a}^{2}}$

In this model the numerical implementation is important to check, after each step of time, whether the noise terms in equations mentioned above have taken $n_{n}, n_{m}$, or $n_{h}$ not inside the range of $[0,1]$. If so, will be repeated and goes to new random numbers for $n_{n}, n_{m}$, or $n_{h}$. (Güler, 2013)

\section{B. The Functions of Noisy Rate}

A typical set of noisy rate functions used by the Hodgkin-Huxley equations is as follows:

$$
\begin{aligned}
& \alpha_{n}=(0.1-0.01 V) /(\exp (1-0.1 V)-1) \\
& \left.\beta_{n}=0.125 \exp (-V / 80)\right) \\
& \alpha_{m}=(2.5-0.1 V) /(\exp (2.5-0.1 V)-1) \\
& \beta_{m}=4 \exp (-V / 18) \\
& \alpha_{h}=0.07 \exp (-V / 20) \\
& \beta_{h}=1 /(\exp (3-0.1 V)+1)
\end{aligned}
$$

The edited the above standard functions as follows:

$$
\begin{aligned}
& \alpha_{n} \leftarrow \alpha_{n}\left(1+\kappa \sin \left(5 V^{n}\right)\right) \\
& \beta_{n} \leftarrow \beta_{n}\left(1-\kappa \sin \left(5 V^{n}\right)\right) \\
& \alpha_{m} \leftarrow \alpha_{m}\left(1-\kappa \sin \left(5 V^{m}\right)\right) \\
& \beta_{m} \leftarrow \beta_{m}\left(1-\kappa \sin \left(5 V^{m}\right)\right) \\
& \alpha_{h} \leftarrow \alpha_{h}\left(1-\kappa \sin \left(5 V^{h}\right)\right) \\
& \beta_{n} \leftarrow \beta_{n}\left(1-\kappa \sin \left(5 V^{h}\right)\right)
\end{aligned}
$$

$\mathrm{V}^{\wedge} \mathrm{n}, \mathrm{V}^{\wedge} \mathrm{m}$, and $\mathrm{V}^{\wedge} \mathrm{h}=$ Ornestein-Uhlenbeck:

$$
\begin{aligned}
& \dot{V}^{n}=50 V^{n}+R^{n} \\
& \dot{V}^{m}=50 V^{m}+R^{m} \\
& \dot{V}^{h}=50 V^{h}+R^{h}
\end{aligned}
$$

$R^{n}, R^{m}$, and $R^{h}=0$ gaussian white noise, so: $\left(R^{n}(t) R^{n}\left(t^{\prime}\right)\right)=\left(R^{m}(t) R^{m}\left(t^{\prime}\right)\right)=\left(R^{h}(t) R^{h}\left(t^{\prime}\right)\right)=$ $100 \delta\left(t-t^{\prime}\right)$

The constants were set to be 50 and 100, respectively in above equations, for convenience. The value $\kappa$ is constant which specifies the strength of the noisy rate, must satisfy: $0 \leq|\kappa| \leq 1$. 


\section{Experiments}

\section{A. Performing Linaro et. al. with computing renormalization solution}

The average spiking frequency and other information shown in Figure 6 and 7 show that the renormalization curve was closed to the Linaro et. al. curve up to the microscopic and microscopic curve at the beginning of the simulation, and the plot after the 6th curve showed that the curve of the Linaro et. al. started to increase close to the microscope curve and unchanged. After reaching the land 10.03, a closer and better renormalization was obtained from the Linaro et. al. model, while the renormalization solution, which was near to the microscope at the start of the simulation, was calculated with $30 \mathrm{sec}$ [1].

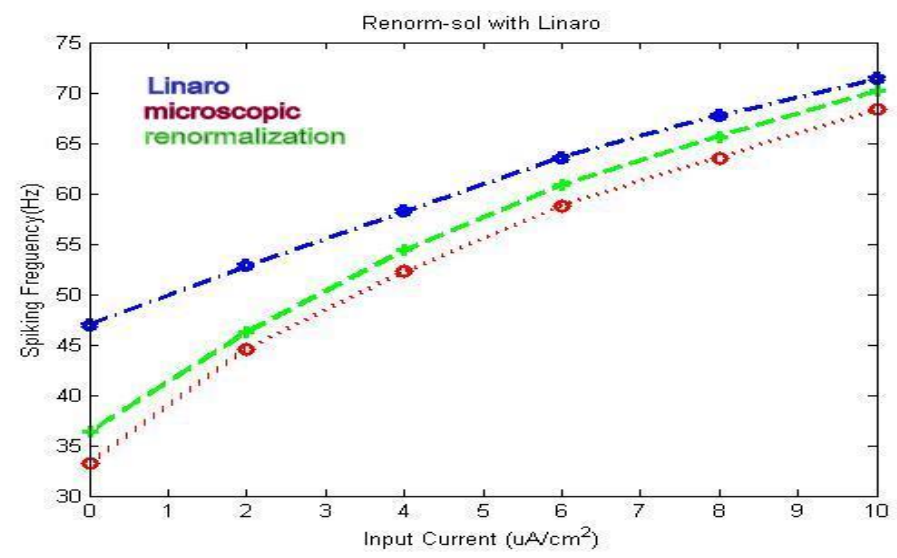

Fig 3: Outcome of amplitude changing between membrane size $(360,1200)$

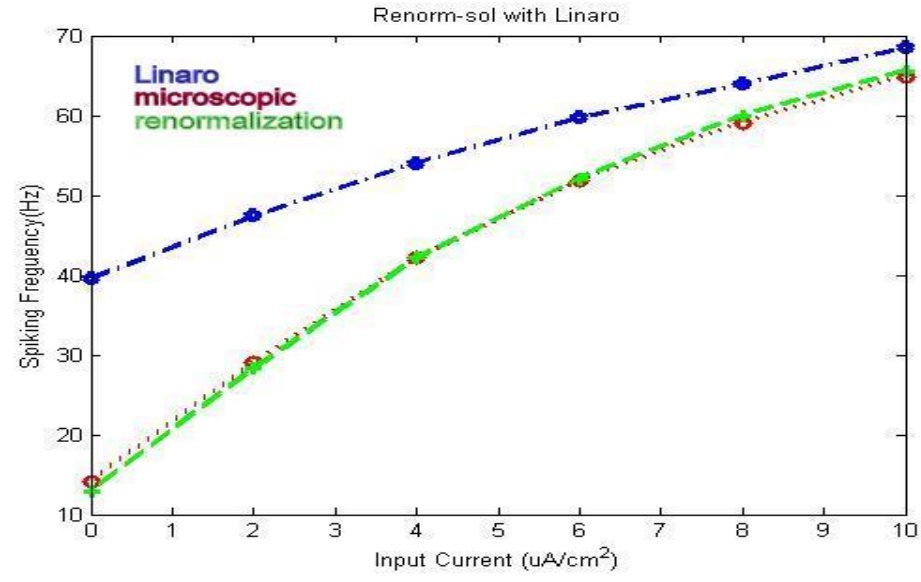

Fig 4: Outcome of amplitude changing between membrane size $(1800,6000)$

\section{B. Response to a Stimulus Pulse}

By using a stimulus pulse the response of model to passing change in the stimulus as shown in Figures $(6,7)$ and a sub-threshold current value, $I=-4{ }_{\mu} \mathrm{A} / \mathrm{cm}^{2}$, was used as the base current. For a $1 \mathrm{~ms}$ of duration, the current swerves from the foundation, arriving some value generated by intensity [1].

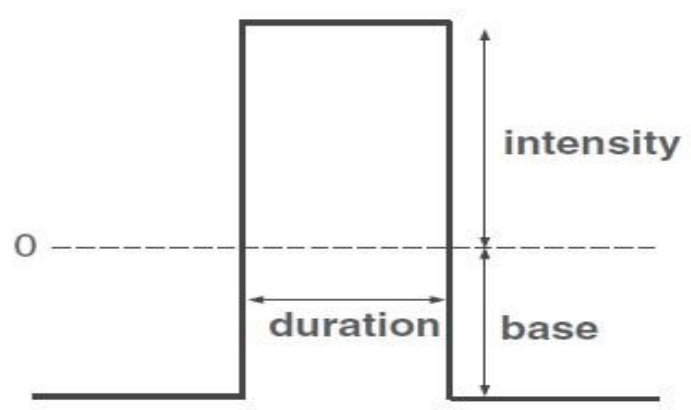

Fig 5: Stimulus Pulse 


\section{Computing Renormalization with Linaro et. al.}

In figures $(3,4)$ shows the renormalization curve is so near to microscopic in start, better than the Linaro et al. curve is far from microscope curve with not changing ploting to 10.03 . So, the renormalization closer the average computed over $30 \mathrm{sec}$.

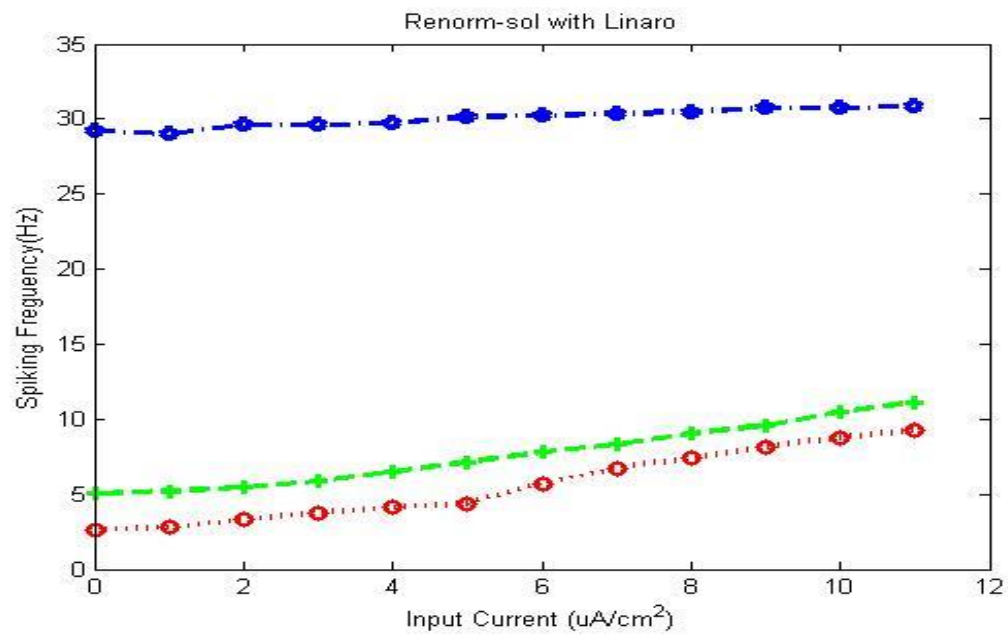

Fig 6: Outcome of amplitude changing between membrane size $(360,1200)$

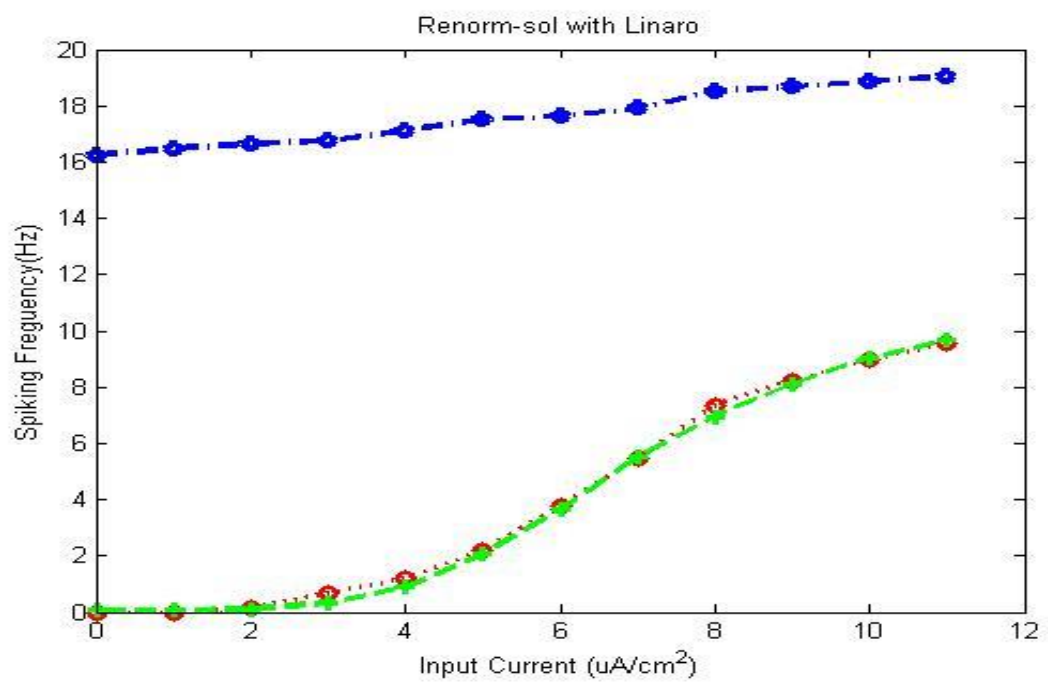

Fig 7: Outcome of amplitude changing between membrane size $(1800,6000)$

\section{Conclusion}

In this paper, we have discussed about the noise of the rate functions and are continuing experiments to examine the Linaro et. al form model perform under that type of spiking, in the different input signals the spiking statistical of noise effected. In an earlier studies (Güler, 2013), was found in the ion channel, a multiplicity of the gates and the key role which will arouse the NCCP and previously found to be the main reason for high excitability of cells and the firing in small size of membrane. They also found that the
NCCP process in promoting a spontaneous firing even the membrane size has big size to activate the noise cell. The paper discussed the NCCP effect on optimization of the coherence spike.

Next, to passing change in the stimulus thar response of model by stimulus pulse shown in figures $(6,7)$ and a sub-threshold current value, $I=-4_{\mu} A / \mathrm{cm}^{2}$, was used as the base current. For a $1 \mathrm{~ms}$ of duration shown in figure 5 . Then in this paper for a set of intensities we computed firing efficiency, latency, and jitter. Shown in Figures $(6,7)$. Each plot in the figures was included by reiterated trials of the corresponding stimulus pulse 
2000 times. As shown in last figures $(6,7)$ the renormalization solution still too close to the microscope with a low frequencies in case of performing Linaro model with steady state of closer model (renormalization solution) to microscope simulation.

\section{References}

[1] Asaad, R. R. (2014). An Investigation of the Neuronal Dynamics Under Noisy Rate Functions (Doctoral dissertation, Eastern Mediterranean University (EMU)-Doğu Akdeniz Üniversitesi (DAÜ)).

[2] Abbot, D. P. (2002). Theoretical Neuroscience Computation and Mathematical Modeling of Neural System. MIT press.

[3] Faisal, A. S. (2008). Noise in the nervous system. nervous system. Nature Reviews Neuroscience, 9, 292-303.

[4] Özer, M. (2006). Frequency-dependent information coding in neurons with stochastic ion channels for subthreshold periodic forcing. Physics Letters A, 354, 258-263.

[5] Sakmann, B. \&. (1995). Single-channel recording (2nded.). New York: Plenum.
[6] Schmid, G. G. (2001). Stochastic resonance as a collective property of ion channel assemblies. Europhysics Letters, 56, 22-28.

[7] Izhikevich, E. M. (2007). Dynamical Systems in Neuroscience:The Geometry of Excitability and Bursting. MIT press.

[8] Jacobson, G. A. (2005). Subthreshold voltage noise of rat neocortical pyramidal neurones. Journal of Physiology, 564,145-160.

[9] Kole, M. H. (2006). Single Ih channels in pyramidal neuron dendrites: Properties, distribution, and impact on action potential output. Journal of Neuroscience, 26, 1677-1687.

[10] Güler, M. (2013). Stochastic Hodgkin-huxley equations with colored noise terms in the conductances. Neural Computation .25:46-74, 2013.

[11] Hodgkin, A. L. (1952). A quantitative description of membrane current and its application to conduction and excitationin in nerve. Journal of Physiology. (London.Print), 117, 500-544.

[12] Whishaw, K. B. (2012). Fundamentals of Human Physiology FOURTH EDITION. Virginia United States. 